\title{
Higher Derivatives and Polynomials of the Standard Nield-Kuznetsov Function of the First Kind
}

\author{
M.H. Hamdan* \\ Department of Mathematics and Statistics \\ University of New Brunswick, Saint John, N.B., E2L 4L5 \\ Canada \\ Email: hamdan@unb.ca \\ S. Jayyousi Dajani \\ Department of Mathematics and Computer Science \\ Lake Forest College, Lake Forest, IL 60045 \\ USA \\ M.S. Abu Zaytoon \\ Department of Mathematics, University of Petra, Amman \\ Jordan
}

Received: June 20, 2021. Received: November 7, 2021. Accepted: November 20, 2021. Published: December 8, 2021.

\begin{abstract}
In this fundamental work, higher derivatives of the standard Nield-Kuznetsov function of the first kind, and the polynomials arising from this function and Airy's functions, are derived and discussed. This work provides background theoretical material and computational procedures for the arising polynomials and the higher derivatives of the recently introduced Nield-Kuznetsov function, which has filled a gap that existed in the literature since the nineteenth century. The ease by which the inhomogeneous Airy's equation can now be solved is an advantage offered by the Nield-Kuznetsov functions. The current analysis might prove to be invaluable in the study of inhomogeneous Schrodinger, Tricomi, and Spark ordinary differential equations.
\end{abstract}

Keywords - Airy's Polynomials, Nield- Kuznetsov Derivatives

\section{INTRODUCTION}

A iry's differential equation, its associated Airy's functions, [1], and extensions of Airy's functions are of importance in the study of circuit theory, systems theory and signal processing since these special functions arise in solutions to Start and Schrodinger equations, (cf. $[2,3,4]$ and the references therein) in addition to their direct applicability in the study of optics, fluid flow and electromagnetism. Many differential equations in mathematical physics and quantum theory can be reduced to Airy's equation by an appropriate change of variables, thus adding to the importance of studies of Airy's functions and their related functions, $[4,5,6]$.

Recently, Airy's equation proved to be a valuable tool in the analysis of flow over porous layers in the presence of a transition layer. Brinkman's equation governs the flow in the variable permeability transition layer, and Nield and Kuznetsov [7] model the flow in such a way that Brinkman's equation reduced to Airy's equation. The same problem was recently analyzed using the generalized Airy's equation, [8].

In their analysis, Nield and Kuznetsov [7] found it convenient to define a new integral function in terms of Airy's functions and their integrals, referred to as the standard Nield-Kuznetsov function of the first kind. The main properties of this newly introduced function were studied by Hamdan and Kamel [9], as it is of great utility in solving the inhomogeneous Airy's equation.

In recent articles, recent developments in analysis of the Nield-Kuznetsov functions were discussed and documented. $[10,11]$ and include methods of computations and solution methodologies to inhomogeneous Airy's and generalized Airy's equations with initial and boundary conditions. Recent work in this field includes pioneering work on the NieldKuznetsov functions and the use of Laplace transform and 
uniform asymptotic expansions [12,13], and to analyze Airy's polynomials that arise when higher derivatives are involved, [14].

Abramochkin and Razueva, [14], provided elegant analysis of Airy's polynomials that arise when one considers higher derivatives of Airy's functions or derivatives of their products. These same polynomials and other polynomials arise in the higher derivatives of the standard Nield-Kuznetsov function of the first kind, and are important from both a theoretical and a practical point of view.

The purpose of this work is to obtain generalizations of the higher derivatives, and polynomials associated with the Standard Nield-Kuznetsov function of the first kind, and introduce their efficient methods of computations. These higher derivatives might find their way in analysis of the Stark equation, Schrodinger equation and Tricomi's inhomogeneous equation.

\section{STANDARD NIELD-KUZNETSOV FUNCTIONS}

Consider the inhomogeneous Airy's ordinary differential equation (ODE)

$y^{\prime \prime}-x y=f(x)$

(1)

wherein prime notation denotes ordinary differentiation with respect to $x$. General solution to (1) depends on the forcing function (right-hand-side of $(1)), f(x)$, for which the scientific literature reports there are five subcases when $f(x)$ is continuous. These are summarized in Table 1, below.

Table 1. Forms of General Solution of Inhomogeneous Airy's

\begin{tabular}{|c|c|}
\hline \multicolumn{2}{|r|}{ Equation } \\
\hline$f(x)$ & General solution of (1) \\
\hline 0 & $\begin{array}{l}y=a_{1} A_{i}(x)+a_{2} B_{i}(x) \\
(2) \\
a_{1}, a_{2} \text { are arbitrary constants }\end{array}$ \\
\hline$f(x)=-\frac{1}{\pi}$ & $\begin{array}{l}y=b_{1} A_{i}(x)+b_{2} B_{i}(x)+G_{i}(x) \\
(3) \\
b_{1}, b_{2} \text { are arbitrary constants }\end{array}$ \\
\hline$f(x)=\frac{1}{\pi}$ & $\begin{array}{l}y=c_{1} A_{i}(x)+c_{2} B_{i}(x)+H_{i}(x) \\
(4) \\
c_{1}, c_{2} \text { are arbitrary constants }\end{array}$ \\
\hline $\begin{array}{l}f(x)=R \\
\text { (Any real } \\
\text { constant) }\end{array}$ & $\begin{array}{l}y=d_{1} A_{i}(x)+d_{2} B_{i}(x)-\pi R N_{i}(x) \\
(5) \\
d_{1}, d_{2} \text { are arbitrary constants }\end{array}$ \\
\hline $\begin{array}{l}f(x) \text { is a } \\
\text { differentiable } \\
\text { function of } x\end{array}$ & $\begin{array}{l}y=e_{1} A_{i}(x)+e_{2} B_{i}(x)+\pi K_{i}(x) \\
-\pi f(x) N_{i}(x) \\
(6) \\
e_{1}, e_{2} \text { are arbitrary constants }\end{array}$ \\
\hline
\end{tabular}

In Table $1, A_{i}(x)$ and $B_{i}(x)$ are two linearly independent functions known as Airy's homogeneous functions of the first and second kind, respectively, and are defined by the following integrals, [4]:

$A_{i}(x)=\frac{1}{\pi} \int_{0}^{\infty} \cos \left(x t+\frac{t^{3}}{3}\right) d t$
$B_{i}(x)=\frac{1}{\pi} \int_{0}^{\infty}\left[\sin \left(x t+\frac{t^{3}}{3}\right)+\exp \left(x t-\frac{t^{3}}{3}\right)\right] d t$

The non-zero Wronskian of $A_{i}(x)$ and $B_{i}(x)$ is given by, [4]:

$W\left(A_{i}(x), B_{i}(x)\right)=A_{i}(x) \frac{d B_{i}(x)}{d x}-B_{i}(x) \frac{d A_{i}(x)}{d x}=\frac{1}{\pi}$

The functions $G_{i}(x)$ and $H_{i}(x)$ are the Scorer functions, [4], given by

$G_{i}(x)=\frac{1}{\pi} \int_{0}^{\infty} \sin \left(x t+\frac{t^{3}}{3}\right) d t$

$H_{i}(x)=\frac{1}{\pi} \int_{0}^{\infty} \exp \left(x t-\frac{t^{3}}{3}\right) d t$

The integral function $N_{i}(x)$ is called the Standard NieldKuznetsov Function of the First Kind, and is given by, [7,9]:

$N_{i}(x)=A_{i}(x) \int_{0}^{x} B_{i}(t) d t-B_{i}(x) \int_{0}^{x} A_{i}(t) d t$

The function $K_{i}(x)$ is called the Standard Nield-Kuznetsov Function of the Second Kind, and takes the following equivalent forms, [9]:

$$
\begin{aligned}
& K_{i}(x)=A_{i}(x) \int_{0}^{x}\left\{\int_{0}^{t} B_{i}(\tau) d \tau\right\} f^{\prime}(t) d t- \\
& B_{i}(x) \int_{0}^{x}\left\{\int_{0}^{t} A_{i}(\tau) d \tau\right\} f^{\prime}(t)
\end{aligned}
$$

$$
\begin{aligned}
& K_{i}(x)=f(x) N_{i}(x)-\left\{A_{i}(x) \int_{0}^{x} f(t) B_{i}(t) d t-\right. \\
& \left.B_{i}(x) \int_{0}^{x} f(t) A_{i}(t) d t\right\} .
\end{aligned}
$$

The integral function $N_{i}(x)$ defined by (12) was introduced in the work of Nield and Kuznetsov [7] in their analysis of the transition layer in the study of flow over porous layers. Hamdan and Kamel [9] showed that the particular solution to Airy's ODE (1) can be expressed in terms of $N_{i}(x)$ when the forcing function $f(x)=R=$ any constant.

Hamdan and Kamel [9] introduced the function $K_{i}(x)$, equations (13) and (14), and showed that the particular solution of Airy's inhomogeneous equation (1) can be represented using $K_{i}(x)$ when the forcing function $f(x)$ is any differentiable function of $x$.

The integral functions $N_{i}(x)$ and $K_{i}(x)$ have been termed the Standard Nield-Kuznetsov functions of the first and second kind, respectively. The term "standard" is used here to distinguish these functions from the Generalized NieldKuznetsov functions of the first and second kind, used to express the particular solutions of the generalized inhomogeneous Airy's equation, [8], and the Parametric Nield-Kuznetsov functions of the first and second kind, introduced in [10] and used to express particular solutions of the inhomogeneous Weber equation. 
The above Nield-Kuznetsov functions possess a large number of mathematically interesting properties and representations, some of which have yet to be studied ( $c f$. [10] and the references therein). Extensions, further applications and properties of the Nield-Kuznetsov functions have also been discussed in [11-13].

In what follows, we discuss some relevant properties of $N_{i}(x)$, its higher derivatives, the polynomials arising as a result of its derivatives, the differential equations it satisfies, its connections to other special functions, and its representations in terms of infinite series to facilitate its computations and computations of its derivatives.

\section{DERIVATIVES OF $N i(x)$}

Abramochkin and Razueva [14] showed that the nth derivative of $A i(x)$ can be expressed in terms of $A i(x)$, $A^{\prime} i(x)$, and some polynomials, $P_{n}(x)$ and $Q_{n}(x)$. Similarly, nth derivative of $B i(x)$ can be expressed in terms of $B i(x)$, $B^{\prime} i(x)$, and the same polynomials, $P_{n}(x)$ and $Q_{n}(x)$. Not surprising, the nth derivative of $N i(x)$ can be expressed in terms of $N i(x), N^{\prime} i(x)$, the same polynomials $P_{n}(x)$ and $Q_{n}(x)$ of [14], and a polynomial $R_{n}(x)$, for $n \geq 2$. This realization was also reported in [9].

The first derivative of $N_{i}(x)$ is obtained by direct differentiation of (12). Clearly, the first derivative is not a polynomial. The second to fifteenth derivatives of $N i(x)$ are polynomials and take the following forms, obtained by repeated differentiation and simplification, with the help of the Wronskian, equation (9). The choice of the first fifteen derivatives has been made to parallel, and compare with, the fifteen derivatives obtained in [14].

$N^{\prime} i(x)=A^{\prime} i(x) \int_{0}^{x} B i(t) d t-B^{\prime} i(x) \int_{0}^{x} A i(t) d t$.

$N^{\prime \prime} i(x)=x N i(x)-\frac{1}{\pi}$

$N^{\prime \prime \prime} i(x)=N i(x)+x N^{\prime} i(x)$

$N^{i v} i(x)=x^{2} N i(x)+2 N^{\prime} i(x)-\frac{x}{\pi}$

$N^{v} i(x)=4 x N i(x)+x^{2} N^{\prime} i(x)-\frac{3}{\pi}$

$N^{v i} i(x)=\left(x^{3}+4\right) N i(x)+6 x N^{\prime} i(x)-\frac{x^{2}}{\pi}$

$N^{v i i} i(x)=9 x^{2} N i(x)+\left(x^{3}+10\right) N^{\prime} i(x)-\frac{8 x}{\pi}$

(21)

$N^{v i i i} i(x)=\left(x^{4}+28 x\right) N i(x)+12 x^{2} N^{\prime} i(x)-\frac{\left(x^{3}+18\right)}{\pi}$

$N^{i x} i(x)=\left(16 x^{3}+28\right) N i(x)+\left(x^{4}+52 x\right) N^{\prime} i(x)-\frac{15 x^{2}}{\pi}$
$N^{x} i(x)=\left(x^{5}+100 x^{2}\right) N i(x)+\left(20 x^{3}+80\right) N^{\prime} i(x)-$

$\frac{\left(x^{4}+82 x\right)}{\pi}$

(24)

$N^{x i} i(x)=\left(25 x^{4}+280 x\right) N i(x)+\left(x^{5}+160 x^{2}\right) N^{\prime} i(x)-$

$\frac{\left(24 x^{3}+162\right)}{\pi}$

(25)

$N^{x i i} i(x)=\left(x^{6}+260 x^{3}+280\right) N i(x)+\left(30 x^{4}+\right.$

$600 x) N^{\prime} i(x)-\frac{\left(x^{5}+232 x^{2}\right)}{\pi}$

(26)

$N^{x i i i} i(x)=\left(36 x^{5}+1380 x^{2}\right) N i(x)+\left(x^{6}+380 x^{3}+\right.$

$880) N^{\prime} i(x)-\frac{\left(35 x^{4}+1064 x\right)}{\pi}$

$N^{x i v} i(x)=\left(x^{7}+560 x^{4}+3640 x\right) N i(x)+\left(42 x^{5}+\right.$

$\left.2520 x^{2}\right) N^{\prime} i(x)-\frac{\left(x^{6}+520 x^{3}+1944\right)}{\pi}$

$N^{x v} i(x)=\left(49 x^{6}+4760 x^{3}+3640\right) N i(x)+\left(x^{7}+\right.$

$\left.770 x^{4}+8680 x\right) N^{\prime} i(x)-\frac{\left(48 x^{5}+4080 x^{2}\right)}{\pi}$

Continuing in this manner, the $n$th derivative of $N i(x)$ can be expressed as:

$N i^{(n)}(x)=P_{n}(x) N i(x)+Q_{n}(x) N^{\prime} i(x)-R_{n}(x) / \pi$

where $P_{n}(x), Q_{n}(x)$ and $R_{n}(x)$ are the polynomial coefficients of $N i(x), N^{\prime} i(x)$ and $W(A i(x), B i(x))$, respectively, in the $n$th derivative of $N i(x)$, for $n \geq 2$. With the knowledge of the $n$th derivative, the $\mathrm{n}+1^{\text {st }}$ derivative can be obtained as:

$N i^{(n+1)}(x)=\left[P^{\prime}{ }_{n}(x)+x Q_{n}(x)\right] N i(x)+\left[P_{n}(x)+\right.$

$\left.Q^{\prime}{ }_{n}(x)\right] N^{\prime} i(x)-\frac{1}{\pi}\left[Q_{n}(x)+R_{n}^{\prime}(x)\right.$

Equation (31) takes the following form in terms of $A i(x)$ and $\operatorname{Bi}(x)$ :

$N^{(n+1)}(x)=\left\{\left[P^{\prime}{ }_{n}(x)+x Q_{n}(x)\right] A i(x)+\left[P_{n}(x)+\right.\right.$ $\left.\left.Q^{\prime}{ }_{n}(x)\right] A^{\prime} i(x)\right\} \int_{0}^{x} B i(t) d t-\left\{\left[P^{\prime}{ }_{n}(x)+x Q_{n}(x)\right] B i(x)+\right.$ $\left.\left[P_{n}(x)+Q_{n}^{\prime}(x)\right] B^{\prime} i(x)\right\} \int_{0}^{x} A i(t) d t-\left[Q_{n}(x)+\right.$ $\left.R^{\prime}(x)\right] W(A i(x), B i(x))$.

Using (30) in (32) yields the $\mathrm{n}+1^{\text {st }}$ derivative as:

$N i^{(n+1)}(x)=P_{n+1}(x) N i(x)+Q_{n+1}(x) N^{\prime} i(x)-\frac{R_{n+1}(x)}{\pi}$

Comparing (31) and (32), establishes the following:

$P_{n+1}(x)=P_{n}^{\prime}(x)+x Q_{n}(x)$ 
$Q_{n+1}(x)=Q^{\prime}{ }_{n}(x)+P_{n}(x)$

(35)

$R_{n+1}(x)=R_{n}^{\prime}(x)+Q_{n}(x)$

(36)

Polynomials, for $n \geq 2$, appearing in the above derivatives can be tabulated as shown in Table 2 .

Table 2. Polynomial Coefficients of $N i(x), N^{\prime} i(x)$ and $W(A i(x), B i(x))$

\begin{tabular}{|l|l|l|l|}
\hline$n$ & \multicolumn{1}{|c|}{$P_{n}(x)$} & \multicolumn{1}{|c|}{$Q_{n}(x)$} & \multicolumn{1}{c|}{$R_{n}(x)$} \\
\hline 2 & $x$ & 0 & 1 \\
\hline 3 & 1 & $x$ & 0 \\
\hline 4 & $x^{2}$ & 2 & $x$ \\
\hline 5 & $4 x$ & $x^{2}$ & 3 \\
\hline 6 & $x^{3}+4$ & $6 x$ & $x^{2}$ \\
\hline 7 & $9 x^{2}$ & $x^{3}+10$ & $8 x$ \\
\hline 8 & $x^{4}+28 x$ & $12 x^{2}$ & $x^{3}+18$ \\
\hline 9 & $16 x^{3}+28$ & $x^{4}+52 x$ & $15 x^{2}$ \\
\hline 10 & $x^{5}+100 x^{2}$ & $20 x^{3}+80$ & $x^{4}+82 x$ \\
\hline 11 & $25 x^{4}+280 x$ & $x^{5}+160 x^{2}$ & $24 x^{3}+162$ \\
\hline 12 & $\begin{array}{l}x^{6}+260 x^{3} \\
+280\end{array}$ & $30 x^{4}+600 x$ & $x^{5}+232 x^{2}$ \\
\hline 13 & $\begin{array}{l}36 x^{5} \\
+1380 x^{2}\end{array}$ & $\begin{array}{l}x^{6}+380 x^{3} \\
+880\end{array}$ & $35 x^{4}+1064 x$ \\
\hline 14 & $\begin{array}{l}x^{7}+560 x^{4} \\
+3640 x\end{array}$ & $\begin{array}{l}42 x^{5} \\
+2520 x^{2}\end{array}$ & $\begin{array}{l}x^{6}+520 x^{3} \\
+1944\end{array}$ \\
\hline 15 & $\begin{array}{l}49 x^{6} \\
+4760 x^{3}\end{array}$ & $\begin{array}{l}x^{7}+770 x^{4} \\
+8680 x\end{array}$ & $\begin{array}{l}48 x^{5} \\
+4080 x^{2}\end{array}$ \\
& +3640 & & \\
\hline
\end{tabular}

The notation used by Abramochkin and Razueva, [14], in that " $n$ " refers to order of the derivative and not the degree of the polynomial is echoed in this work. In fact, degrees of these polynomials are provided in the following Table 3 .

Table 3. Coefficient Polynomial Degrees

\begin{tabular}{|c|l|l|}
\hline Polynomial & $\begin{array}{l}\text { Degree } \\
\text { (when } n \text { is even) }\end{array}$ & $\begin{array}{l}\text { Degree } \\
\text { (when } n \text { is odd) }\end{array}$ \\
\hline$P_{n}(x)$ & $\frac{n}{2} ; n \geq 2$ & $\frac{n-3}{2} ; n \geq 3$ \\
\hline$Q_{n}(x)$ & $\frac{n}{2}-2 ; n \geq 4$ & $\frac{n-1}{2} ; n \geq 3$ \\
\hline$R_{n}(x)$ & $\frac{n}{2}-1 ; n \geq 2$ & $\frac{n-5}{2} ; n \geq 5$ \\
\hline
\end{tabular}

A. Values of Polynomials and Derivatives of Ni(x) at $x=0$

Evaluating (30) at $x=0$, gives

$N i^{(n)}(0)=P_{n}(0) N i(0)+Q_{n}(0) N^{\prime} i(0)-\frac{R(0)}{\pi}$

(37)

Equations (12) and (15) provide $N i(0)=0$ and $N^{\prime} i(0)=0$, and (36) yields
$N i^{(n)}(0)=-\frac{R(0)}{\pi}$

In addition, using (32), gives

$N i^{(n+1)}(0)=-\frac{R_{n+1}(0)}{\pi}=-\frac{\left[Q_{n}(0)+R^{\prime}{ }_{n}(0)\right]}{\pi}$

The following recursive relation can also be established.

$N i^{(n)}(0)=(n-2) N i^{(n-3)}(0)$ for $n=3,4, \quad 5, \ldots$ (40)

$N i^{(n+1)}(0)=(n-1) N i^{(n-2)}(0)$ for $n=2,3,4, \ldots$ (41)

Values of polynomials $P_{n}(x), Q_{n}(x)$, and $R_{n}(x)$ and derivatives of $N i(x)$ at $x=0$ are shown in the following Table 4.

Table 4. Values of Coefficient Polynomials and Derivatives of $N i(x)$ at $x=0$

\begin{tabular}{|l|l|l|l|l|}
\hline$n$ & $P_{n}(0)$ & $Q_{n}(0)$ & $R_{n}(0)$ & \multicolumn{1}{|c|}{$i^{(n)}(0)$} \\
\hline 2 & 0 & 0 & 1 & $-\frac{1}{\pi}$ \\
\hline 3 & 1 & 0 & 0 & 0 \\
\hline 4 & 0 & 2 & 0 & 0 \\
\hline 5 & 0 & 0 & 3 & $-\frac{3}{\pi}$ \\
\hline 6 & 4 & 0 & 0 & 0 \\
\hline 7 & 0 & 10 & 0 & 0 \\
\hline 8 & 0 & 0 & 18 & $-\frac{18}{\pi}$ \\
\hline 9 & 28 & 0 & 0 & 0 \\
\hline 10 & 0 & 80 & 0 & 0 \\
\hline 11 & 0 & 0 & 162 & $-\frac{162}{\pi}$ \\
\hline 12 & 280 & 0 & 0 & 0 \\
\hline 13 & 0 & 880 & 0 & 0 \\
\hline 14 & 0 & 0 & 1944 & $-\frac{1944}{\pi}$ \\
\hline 15 & 3640 & 0 & 0 & 0 \\
\hline
\end{tabular}

\section{B. Derivatives of $\mathrm{Ni}(x)$ in Terms of Bessel Functions}

With the knowledge of the expressions of $A_{i}(x)$ and $B_{i}(x)$, their first derivatives and integrals in terms of Bessel's function of the first kind as, [4]:

$A_{i}(x)=\frac{\sqrt{x}}{3}\left[I_{-\frac{1}{3}}(\mu)-I_{\frac{1}{3}}(\mu)\right]$

$A^{\prime}{ }_{i}(x)=-\frac{x}{3}\left[I_{-\frac{2}{3}}(\mu)-I_{\frac{2}{3}}(\mu)\right]$ 
$B_{i}(x)=\sqrt{\frac{x}{3}}\left[I_{-\frac{1}{3}}(\mu)+I_{\frac{1}{3}}(\mu)\right]$

$B^{\prime}{ }_{i}(x)=\frac{x}{\sqrt{3}}\left[I_{-\frac{2}{3}}(\mu)+I_{\frac{2}{3}}(\mu)\right]$

$\int_{0}^{x} A_{i}(t) d t=\frac{1}{3} \int_{0}^{\mu}\left[I_{-\frac{1}{3}}(t)-I_{\frac{1}{3}}(t)\right] d t$

$\int_{0}^{x} B_{i}(t) d t=\frac{1}{\sqrt{3}} \int_{0}^{\mu}\left[I_{-\frac{1}{3}}(t)+I_{\frac{1}{3}}(t)\right] d t$

where $\mu=\frac{2}{3} x^{3 / 2}$. The function $N i(x)$, its fist derivative, and its higher derivatives, can be expressed in terms of Bessel's function, as

$N i(x)=\frac{2 \sqrt{x}}{3 \sqrt{3}}\left[I_{-\frac{1}{3}}(\mu) \cdot \int_{0}^{\mu} I_{\frac{1}{3}}(t) d t-I_{\frac{1}{3}}(\mu) \int_{0}^{\mu} I_{-\frac{1}{3}}(t) d t\right]$

$N^{\prime} i(x)=\frac{2 x}{3 \sqrt{3}}\left[I_{\frac{2}{3}}(\mu) \cdot \int_{0}^{\mu} I_{\frac{1}{3}}(t) d t-I_{-\frac{2}{3}}(\mu) \cdot \int_{0}^{\mu} I_{-\frac{1}{3}}(t) d t\right]$

$N i^{(n)}(x)=P_{n}(x) \frac{2 \sqrt{x}}{3 \sqrt{3}}\left\{I_{-\frac{1}{3}}(\mu) \cdot \int_{0}^{\mu} I_{\frac{1}{3}}(t) d t-\right.$

$\left.\left.I_{\frac{1}{3}}(\mu) \int_{0}^{\mu} I_{-\frac{1}{3}}(t) d t\right]\right\}+Q_{n}(x) \frac{2 x}{3 \sqrt{3}}\left\{I_{\frac{2}{3}}(\mu) \cdot \int_{0}^{\mu} I_{\frac{1}{3}}(t) d t-\right.$

$\left.I_{-\frac{2}{3}}(\mu) \cdot \int_{0}^{\mu} I_{-\frac{1}{3}}(t) d t\right\}-\frac{R_{n}(x)}{\pi}$

$(50)$

$N i^{(n+1)}(x)=\left[P^{\prime}{ }_{n}(x)+x Q_{n}(x)\right] \frac{2 \sqrt{x}}{3 \sqrt{3}}\left\{I_{-\frac{1}{3}}(\mu) \cdot \int_{0}^{\mu} I_{\frac{1}{3}}(t) d t-\right.$

$\left.\left.I_{\frac{1}{3}}(\mu) \int_{0}^{\mu} I_{-\frac{1}{3}}(t) d t\right]\right\}+\left[P_{n}(x)+\right.$

$\left.Q^{\prime}{ }_{n}(x)\right] \frac{2 x}{3 \sqrt{3}}\left\{I_{\frac{2}{3}}(\mu) \cdot \int_{0}^{\mu} I_{\frac{1}{3}}(t) d t-I_{-\frac{2}{3}}(\mu) \cdot \int_{0}^{\mu} I_{-\frac{1}{3}}(t) d t\right\}-$

$\frac{1}{\pi}\left[Q_{n}(x)+R^{\prime}{ }_{n}(x)\right.$

(51)

\section{Differential Equations that $\mathrm{Ni}(x)$ Satisfies}

The function $N_{i}(x)$ satisfies the following nth order ordinary differential equations, obtained from (30):

$y^{(n)}-Q_{n}(x) y^{\prime}-P_{n}(x) y=-R_{n}(x) / \pi$

For instance, $N i(x)$ is a solution of the following second- and third-order equations, respectively:

$y^{\prime \prime}-x y=-1 / \pi$

(53)

$y^{\prime \prime \prime}-x y^{\prime}-y=0$
It is worth noting that (54) is a special case of the wellknown comparison ordinary differential equation that received interest in the literature (see [4], page 108, and Langer, [15]), namely:

$y^{\prime \prime \prime}-x y^{\prime}-\lambda y=0$.

When $\lambda=1$, equation (55) reduces to (54). Solutions to (55) have been discussed in detail in [4]. However, it is seen here that $\mathrm{Ni}(x)$ is a solution to (55) for the case of $\lambda=1$. Furthermore, as discussed in [4], if $y$ is a solution of (55) then $z=y^{\prime}$ is a solution of the equation:

$z^{\prime \prime \prime}-x z^{\prime}-(1+\mu) z=0$.

Accordingly, since the function $y=N i(x)$ is a solution to (54), the function $z=N i^{\prime}(x)$ is a solution of

$z^{\prime \prime \prime}-x z^{\prime}-2 z=0$.

\section{REPRESENTATIONS OF $N i(x)$ AND $N^{\prime} i(x)$}

\section{A. Series Representations and Computation of $\mathrm{Ni}(x)$ and $N^{\prime} i(x)$}

Computing and evaluating $N_{i}(x)$ and $N^{\prime}{ }_{i}(x)$ are necessary in solving initial and boundary value problems involving the inhomogeneous Airy's equations and in evaluating higher derivatives of $N_{i}(x)$, which are expressed in terms of $N_{i}(x)$ and $N^{\prime}{ }_{i}(x)$. Typically, Airy's functions are expressed in terms of asymptotic or ascending series that provide approximations to these functions at given values of $x$. Since $N_{i}(x)$ is defined in terms of Airy's functions, we will rely on their approximations to express $N_{i}(x)$ in terms of asymptotic and ascending series for $A_{i}(x)$ and $B_{i}(x)$, their derivatives and integrals.

\section{B. Asymptotic approximations}

The functions $N_{i}(x)$ and $N_{i}^{\prime}(x)$ can be expressed using the following asymptotic series approximations, given in [12], wherein $\mu=\frac{2}{3} x^{3 / 2}$ :

$A_{i}(x) \approx \frac{\exp (-\mu)}{2 \sqrt{\pi} x^{\frac{1}{4}}}\left[1+\frac{(3)(5)}{1 !(-216 \mu)}+\frac{5(7)(9)(11)}{2 !(-216 \mu)^{2}}+\cdots\right]$

$B_{i}(x) \approx \frac{\exp (\mu)}{\sqrt{\pi} x^{\frac{1}{4}}}\left[1+\frac{(3)(5)}{1 !(216 \mu)}+\frac{5(7)(9)(11)}{2 !(216 \mu)^{2}}+\cdots\right]$

$A^{\prime}{ }_{i}(x) \approx-\frac{x^{\frac{1}{4}} \exp (-\mu)}{2 \sqrt{\pi}}\left[1-\frac{(3)(7)}{1 !(-216 \mu)}-\frac{5(7)(9)(13)}{2 !(-216 \mu)^{2}}+\cdots\right]$

$B^{\prime}{ }_{i}(x) \approx \frac{x^{\frac{1}{4}} \exp (\mu)}{\sqrt{\pi}}\left[1-\frac{(3)(7)}{1 !(216 \mu)}-\frac{5(7)(9)(13)}{2 !(216 \mu)^{2}}+\cdots\right]$ 
$\int_{0}^{x} A_{i}(t) d t \approx \frac{1}{3}-\frac{\exp (-\mu)}{2 \sqrt{\pi} x^{\frac{3}{4}}}\left[1-\frac{41}{48 x^{\frac{3}{2}}}+\frac{9241}{4608 x^{2}}+\cdots\right]$

$\int_{0}^{x} B_{i}(t) d t \approx \frac{\exp (\mu)}{\sqrt{\pi} x^{\frac{3}{4}}}\left[1+\frac{41}{48 x^{\frac{3}{2}}}+\frac{9241}{4608 x^{2}}+\cdots\right]$

Substituting (58)-(63) in (12) and (15), we obtain:

$N_{i}(x) \approx \frac{1}{2 \pi x^{2}}-\frac{1}{3} B i(x)=\frac{1}{2 \pi x^{2}}-\frac{\exp (\mu)}{3 \sqrt{\pi} x^{1 / 4}}$

$N^{\prime}{ }_{i}(x) \approx-\frac{1}{2 \pi x^{3 / 2}}-\frac{1}{3} \mathrm{~B} i^{\prime}(x)=-\frac{1}{2 \pi x^{3 / 2}}-\frac{x^{1 / 4} \exp (\mu)}{3 \sqrt{\pi}}$

For large $x$, expressions (64) and (65) take the following forms:

$N_{i}(x) \approx-\frac{1}{3} B i(x)=-\frac{\exp (\mu)}{3 \sqrt{\pi} x^{1 / 4}}$

$N^{\prime}{ }_{i}(x) \approx-\frac{1}{3} \mathrm{Bi}^{\prime}(x)=-\frac{x^{1 / 4} \exp (\mu)}{3 \sqrt{\pi}}$

Using (66) and (67) in (30) and (31), we obtain the following expressions for the $n$th and $n+1^{\text {st }}$ derivatives, respectively, that are valid for large values of $x$ :

$N_{i}^{(n)}(x)=-\frac{\exp (\mu)}{3 \sqrt{\pi} x^{1 / 4}} P_{n}(x)-\frac{x^{1 / 4} \exp (\mu)}{3 \sqrt{\pi}} Q_{n}(x)-R_{n}(x) / \pi$

$N_{i}^{(n+1)}(x)=-\frac{\exp (\mu)}{3 \sqrt{\pi} x^{1 / 4}}\left[P^{\prime}{ }_{n}(x)+x Q_{n}(x)\right]-$

$\frac{x^{1 / 4} \exp (\mu)}{3 \sqrt{\pi}}\left[P_{n}(x)+Q^{\prime}{ }_{n}(x)\right]-\frac{1}{\pi}\left[Q_{n}(x)+R_{n}^{\prime}(x)\right.$

(69)

\section{Ascending Series Representation:}

In order to develop ascending series representations for $N_{i}(x)$ and $N^{\prime}{ }_{i}(x)$, we employ the ascending series representations of the Airy functions, their derivatives and integrals, reported in [4], using Airy's atoms, as follows, wherein $F^{\prime}=f$ :

$A_{i}(x)=a_{1}{F^{\prime}}_{1}(x)-a_{2} F_{2}^{\prime}(x)$

(70)

$B_{i}(x)=\sqrt{3} a_{1} F^{\prime}{ }_{1}(x)+\sqrt{3} a_{2} F^{\prime}{ }_{2}(x)$

(71)

$A^{\prime}{ }_{i}(x)=a_{1} F^{\prime \prime}{ }_{1}(x)-a_{2} F^{\prime \prime}{ }_{2}(x)$

(72)

$B_{i}^{\prime}(x)=\sqrt{3} a_{1} F^{\prime \prime}{ }_{1}(x)+\sqrt{3} a_{2} F^{\prime \prime}{ }_{2}(x)$

(73) $\int_{0}^{x} A_{i}(t) d t=a_{1} F_{1}(x)-a_{2} F_{2}(x)$

(74)

$\int_{0}^{x} B_{i}(x) d t=\sqrt{3} a_{1} F_{1}(x)+\sqrt{3} a_{2} F_{2}(x)$

(75)

where

$a_{1}=A_{i}(0)=\frac{1}{3^{2 / 3} \Gamma\left(\frac{2}{3}\right)}$

$a_{2}=-A^{\prime}{ }_{i}(0)=\frac{1}{3^{1 / 3} \Gamma\left(\frac{1}{3}\right)}$

$F_{1}(x)=\sum_{k=0}^{\infty}\left(\frac{1}{3}\right)_{k} \frac{3^{k} x^{3 k+1}}{(3 k+1) !}$

$F^{\prime}{ }_{1}(x)=\sum_{k=0}^{\infty}\left(\frac{1}{3}\right)_{k} \frac{3^{k} x^{3 k}}{(3 k) !}$

$F^{\prime \prime}{ }_{1}(x)=\sum_{k=0}^{\infty}\left(\frac{1}{3}\right)_{k} \frac{3^{k} x^{3 k-1}}{(3 k-1) !}$

$F_{2}(x)=\sum_{k=0}^{\infty}\left(\frac{2}{3}\right)_{k} \frac{3^{k} x^{3 k+2}}{(3 k+2) !}$

$F^{\prime}{ }_{2}(x)=\sum_{k=0}^{\infty}\left(\frac{2}{3}\right)_{k} \frac{3^{k} x^{3 k+1}}{(3 k+1) !}$

$F^{\prime \prime}{ }_{2}(x)=\sum_{k=0}^{\infty}\left(\frac{2}{3}\right)_{k} \frac{3^{k} x^{3 k}}{(3 k) !}$

and $(b)_{k}$ is the Pochhammer symbol, defined by, [4]:

$(b)_{k}=\frac{\Gamma(b+k)}{\Gamma(b)}=b(b+1)(b+2) \ldots(b+k-1) ;(b)_{0}=1$.

Using (70)-(75) in (12), yields

$N i(x)=2 \sqrt{3} a_{1} a_{2}\left\{F_{2} F_{1}^{\prime}-F_{1} F_{2}^{\prime}\right\}$

Differentiating (85) gives

$N^{\prime} i(x)=2 \sqrt{3} a_{1} a_{2}\left\{F_{2} F^{\prime \prime}{ }_{1}-F_{1} F^{\prime \prime}{ }_{2}\right\}$

Upon substituting (76)-(84), the following series are obtained for $N_{i}(x)$ and $N_{i}^{\prime}(x)$ : 


$$
\begin{aligned}
& N_{i}(x)=2 \sqrt{3} a_{1} a_{2}\left[\left\{\sum_{k=0}^{\infty}\left(\frac{1}{3}\right)_{k} \frac{3^{k} x^{3 k}}{(3 k) !}\right\}\left\{\sum_{k=0}^{\infty}\left(\frac{2}{3}\right)_{k} \frac{3^{k} x^{3 k+2}}{(3 k+2) !}\right\}-\right. \\
& \left.\left\{\sum_{k=0}^{\infty}\left(\frac{2}{3}\right)_{k} \frac{3^{k} x^{3 k+1}}{(3 k+1) !}\right\}\left\{\sum_{k=0}^{\infty}\left(\frac{1}{3}\right)_{k} \frac{3^{k} x^{3 k+1}}{(3 k+1) !}\right\}\right]
\end{aligned}
$$

$N^{\prime}{ }_{i}(x)=$

$2 \sqrt{3} a_{1} a_{2}\left[\left\{\sum_{k=0}^{\infty}\left(\frac{1}{3}\right)_{k} \frac{3^{k} x^{3 k-1}}{(3 k-1) !}\right\}\left\{\sum_{k=0}^{\infty}\left(\frac{2}{3}\right)_{k} \frac{3^{k} x^{3 k+2}}{(3 k+2) !}\right\}-\right.$

$\left.\left\{\sum_{k=0}^{\infty}\left(\frac{2}{3}\right)_{k} \frac{3^{k} x^{3 k}}{(3 k) !}\right\}\left\{\sum_{k=0}^{\infty}\left(\frac{1}{3}\right)_{k} \frac{3^{k} x^{3 k+1}}{(3 k+1) !}\right\}\right]$

Equations (87) and (88) can be expressed in the following form when use is made of the definition of Cauchy product:

$N_{i}(x)=$

$2 \sqrt{3} a_{1} a_{2} \sum_{k=0}^{\infty} 3^{k}\left\{\sum_{l=0}^{k}\left(\frac{1}{3}\right)_{l}\left(\frac{2}{3}\right)_{k-l}\left(\frac{-3 k+6 l-1}{(3 l+1) !(3(k-l)+2) !}\right)\right\} x^{3 k+2}$

$N^{\prime}{ }_{i}(x)=2 \sqrt{3} a_{1} a_{2} \sum_{k=0}^{\infty} 3^{k}(3 k+$

2) $\left\{\sum_{l=0}^{k}\left(\frac{1}{3}\right)_{l}\left(\frac{2}{3}\right)_{k-l}\left(\frac{-3 k+6 l-1}{(3 l+1) !(3(k-l)+2) !}\right)\right\} x^{3 k+1}$

Using (89) and (90) in (30) and (31) yields

$N_{i}^{(n)}(x)=2 \sqrt{3} a_{1} a_{2} P_{n}(x) *$

$\sum_{k=0}^{\infty} 3^{k}\left\{\sum_{l=0}^{k}\left(\frac{1}{3}\right)_{l}\left(\frac{2}{3}\right)_{k-l}\left(\frac{-3 k+6 l-1}{(3 l+1) !(3(k-l)+2) !}\right)\right\} *$

$x^{3 k+2}+2 \sqrt{3} a_{1} a_{2} Q_{n}(x) \sum_{k=0}^{\infty} 3^{k}(3 k+$

2) $\left\{\sum_{l=0}^{k}\left(\frac{1}{3}\right)_{l}\left(\frac{2}{3}\right)_{k-l}\left(\frac{-3 k+6 l-1}{(3 l+1) !(3(k-l)+2) !}\right)\right\} x^{3 k+1}-R_{n}(x) / \pi$

$N_{i}^{(n+1)}(x)=2 \sqrt{3} a_{1} a_{2}\left[P^{\prime}{ }_{n}(x)+x Q_{n}(x)\right]$

$\sum_{k=0}^{\infty} 3^{k}\left\{\sum_{l=0}^{k}\left(\frac{1}{3}\right)_{l}\left(\frac{2}{3}\right)_{k-l}\left(\frac{-3 k+6 l-1}{(3 l+1) !(3(k-l)+2) !}\right)\right\} x^{3 k+2}+$

$2 \sqrt{3} a_{1} a_{2}\left[P_{n}(x)+Q^{\prime}{ }_{n}(x)\right] \sum_{k=0}^{\infty} 3^{k}(3 k+$

2) $\left\{\sum_{l=0}^{k}\left(\frac{1}{3}\right)_{l}\left(\frac{2}{3}\right)_{k-l}\left(\frac{-3 k+6 l-1}{(3 l+1) !(3(k-l)+2) !}\right)\right\} x^{3 k+1}-\frac{1}{\pi}\left[Q_{n}(x)+\right.$ $\left.R^{\prime}{ }_{n}(x)\right]$

(92)

\section{CONCLUSION}

In this work, a fundamental framework in which efficient expressions for the nth derivative of the standard NieldKuznetsov function of the first kind are obtained and represented using ascending and asymptotic series has been presented. The framework was achieved through generalizations of the associated coefficient polynomials and using them in the computational series expressions of the nth derivative. Expressions for the $n t h$ derivative of $N i(x)$ in terms of Bessel functions have also been obtained.

Although this work is fundamental in nature, it is hoped that future research will show some value of the knowledge of the $n t h$ derivative of $N i(x)$ and its associated polynomials in obtaining and computing solutions to Schrodinger, Stark and Tricomi inhomogeneous equations.
References

[1] G.B. Airy, "On the intensity of light in the neighbourhood of a caustic", Trans. Cambridge Phil. Soc. vol. 6, pp. 379401,1838

[2] L. Farhang Matin, H. Hasan Bouzari and F. Ahmadi, "Solving Schrodinger equation specializing to the Stark effect in linear potential by the canonical function method", J Theor Appl Phys, vol. 8(3), 6 pp., 2014.

[3] A.Kh. Khanmamedov, M.G. Makhmudoval and N.F Gafarova, "Special Solutions of the Stark equation", Advanced Mathematical Models \& Applications, vol. 6(1), No.1, pp. 59-62, 2021

[4] O. Vallée and M. Soares, Airy functions and applications to physics. World Scientific, London, 2004.

[5] N.M. Temme. Special functions: An introduction to the classical functions of mathematical physics. John Wiley \& Sons, New York, 1996.

[6] M. Abramowitz and I.A. Stegun, Handbook of Mathematical Functions, Dover, New York 1984.

[7] D.A. Nield and A.V. Kuznetsov, "The effect of a transition layer between a fluid and a porous medium: shear flow in a channel", Transport in Porous Media, vol. 78, pp. 477-487, 2009.

[8] M. S. Abu Zaytoon, T. L. Alderson and M. H. Hamdan, "Flow through a variable permeability Brinkman porous core", J. Appl. Mathematics and physics, vol. 4, pp. 766778, 2016.

[9] M.H. Hamdan and M.T. Kamel, "On the Ni(x) integral function and its application to the Airy's non homogeneous equation", Applied Math. Comput., vol. 21 No. 17 , pp. 7349-7360, 2011 .

[10] M.H. Hamdan, S. Jayyousi Dajani and M,S. Abu Zaytoon, "Nield-Kuznetsov functions: Current advances and new results", Int. J. CIRCUITS, SYSTEMS AND SIGNAL PROCESSING, vol. 15, pp. 1506-1520, 2021.

[11] M.H. Hamdan, S.M. Alzahrani, M.S. Abu Zaytoon and S. Jayyousi Dajani, "Inhomogeneous Airy's and generalized Airy's equations with initial and boundary conditions", Int. J. CIRCUITS, SYSTEMS AND SIGNAL PROCESSING, vol. 15, pp. 1486-1496, 2021.

[12] T.M. Dunster, "Uniform asymptotic expansions for solutions of the parabolic cylinder and Weber equations", J. Classical Analysis, vol. 17, No. 1, pp. 69-107, 2021.

[13] T.M. Dunster, "Nield-Kuzenetsov functions and Laplace transforms of parabolic cylinder functions", SIAM J. Math. Anal.vol. 53 No. 5, 2021, pp. 5915-5947, 2021.

[13] E.G. Abramochkin and E.V. Razueva, "Higher derivatives of Airy's functions and of their products", SIGMA, vol. 14, pp. 1-26, 2018.

[14] R.E. Langer, "The solutions of the differential equation $\mathrm{v}^{\prime \prime \prime}+\lambda^{2} \mathrm{w}^{\prime}+3 \mu \lambda^{2} \mathrm{v}=0$ ", Duke Math. vol. 22, pp. 525541,1955 .

\section{Contribution of Individual Authors to the Creation of a Scientific Article (Ghostwriting Policy)}

All authors participated in literature review and problem formulation, and designed plan of the work. All three authors independently obtained the derivatives and polynomials.

M.H. Hamdan organized and wrote the manuscript.

\section{Sources of Funding for Research Presented in a Scientific Article or Scientific Article Itself}

No funding has been received for this work.

Creative Commons Attribution License 4.0 (Attribution 4.0 International, CC BY 4.0)

This article is published under the terms of the Creative Commons Attribution License 4.0

https://creativecommons.org/licenses/by/4.0/deed.en US 